Utah State University

DigitalCommons@USU

2018

\title{
Effects of Sample Adhesives Acoustic Properties on Spatial Resolution of Pulsed Electroacoustic Measurements
}

Zachary Gibson

JR Dennison

Utah State Univesity

Lee Pearson

Box Elder Innovations, LLC

Erick Griffiths

Box Elder Innovations LLC

Anthony Pearson

Box Elder Innovations LLC

Virginie Griseri

Universite de Toulouse

Follow this and additional works at: https://digitalcommons.usu.edu/mp_conf

Part of the Condensed Matter Physics Commons

\section{Recommended Citation}

Gibson, Zachary; Dennison, JR; Pearson, Lee; Griffiths, Erick; Pearson, Anthony; and Griseri, Virginie, "Effects of Sample Adhesives Acoustic Properties on Spatial Resolution of Pulsed Electroacoustic Measurements" (2018). CEIDP. Conference Proceedings. Paper 48.

https://digitalcommons.usu.edu/mp_conf/48

This Report is brought to you for free and open access by the Materials Physics at DigitalCommons@USU. It has been accepted for inclusion in Conference Proceedings by an authorized administrator of DigitalCommons@USU. For more information, please contact digitalcommons@usu.edu.

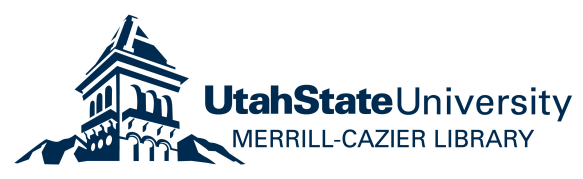




\title{
Effects of Sample Adhesives Acoustic Properties on Spatial Resolution of Pulsed Electroacoustic Measurements
}

\author{
Zachary Gibson ${ }^{1}$, JR Dennison $^{1}$, Lee Pearson $^{2}$, Erick Griffiths $^{2}$, Anthony Pearson $^{2}$, Virginie Griseri ${ }^{3}$ \\ ${ }^{1}$ Utah State University Dept. of Physics, 4415 Old Main Hill, Logan, UT 84322-4415, USA \\ ${ }^{2}$ Box Elder Innovations LLC, Corinne, UT, USA \\ ${ }^{3}$ Université de Toulouse, UPS, INP, LAPLACE (Laboratoire Plasma et Conversion d'Energie)
}

\begin{abstract}
The media chosen to couple the PEA stack (electrode/sample/sensor/backing) can affect the spatial resolution and shape of the response from a Pulsed Electroacoustic (PEA) system significantly. The PEA stack layers must be electrically and acoustically coupled to optimize the amplitude, quality, and spatial resolution of the PEA measurements. Various coupling layer materials were used with $250 \mu \mathrm{m}$ thick polymethylmethacrylate (PMMA) samples and a standard $\sim \mathbf{1 0} \mu \mathrm{m}$ thick PVDF sensor. Coupling layers tested in this study include no media (with substantial pressure applied), light machine oil, silicone oil, and cyanoacrylate (super glue). Pulse amplitudes of $2000 \mathrm{~V}$ and $5 \mathrm{~ns}$ width were used. Static $8 \mathrm{kV}$ DC bias was applied to the sample in order to detect a signal, as the samples were initially free of charge, and to see the interfaces more clearly and showcase the differences in response from the various coupling media. The best option was found to be a single layer of cyanoacrylate at the ground electrodesample interface; this is the only viable option for in vacuo PEA measurements of the media tested.
\end{abstract}

\section{INTRODUCTION}

Pulsed Electroacoustic (PEA) measurements are arguably the most promising of several methods for making measurements of spatial distributions and time evolution of embedded charge [1,2]. Such measurements have many important applications. These include studies of enhanced spacecraft materials and the increased survivability of spacecraft systems in extreme space environments. They also have widespread applications, including microelectronics, high-power electronic devices, high-voltage DC power cable insulation, high-energy accelerators, plasma physics facilities and deposition systems, and microwave generators [1]. Advantages of PEA method include nondestructive measurements, low cost, simplified modeling, and high spatial resolution typically on the order of $10 \mu \mathrm{m}$ [2-4].

The PEA method, outlined in Fig. 1, is as follows [1]. An electric field is applied to a charged layer within a dielectric with a pulsed high voltage signal. This produces an electric force on the embedded charge, creating a pressure (acoustic) wave pulse that propagates through the material and can then be detected by a piezoelectric transducer. Simple time-of-flight analysis determines the position of a thin charge layer; more complex distributions of charge can be studied with more complex analysis. In many current systems, including the custom PEA system used here [5], the dielectric is clamped between rigid thick conducting cathode and anode electrodes. The PEA stack must be electrically and acoustically coupled at each interface between cathode, sample, anode, sensor, and absorber/backing (see Fig. 1).

The choice of coupling media at these interfaces is nontrivial. There can be many adverse effects of the coupling media on the measured PEA waveform. If a coupling media has electrical conductivity comparable to the sample material, the electric field strength across the sample is diminished and harder to determine, thereby reducing the PEA signal strength and signal-to-noise ratio. Lower viscosity coupling layers can flow to unwanted areas, causing contamination and electric field issues. High conductivity, low viscosity materials can cause parallel electrical paths around the sample resulting in a short. Regardless of conductivity, a thick or acoustically mismatched coupling layer will cause multiple acoustic reflections within the coupling media, thereby adding ringing to the resulting waveform. Nonuniform, poorly known thicknesses,
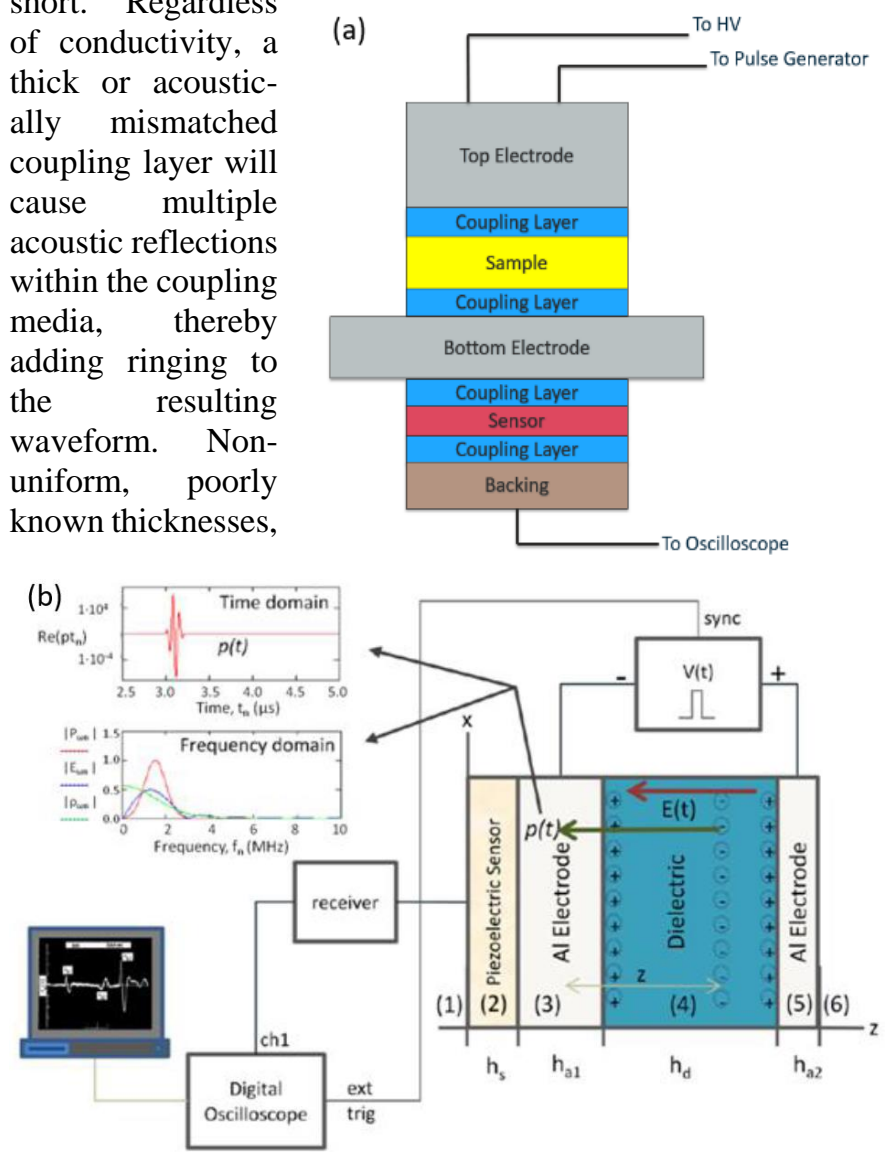

Fig. 1. (a) PEA stack schematic emphasizing the coupling layers in the PEA stack. (b) Block diagram of a typical PEA system. Dielectric material is placed in contact with the cathode and anode conducting electrodes. A signal generator produces a pulsed electric field. This causes acoustic pressure waves, which are detected by a piezoelectric sensor, and recorded on a storage oscilloscope. Signal processing is used to obtain charge distribution plots [1]. 
Table 1. Relevant Material Properties

$\begin{array}{lccc} & \text { Light Machine Oil } & \text { Silicone Oil } & \text { Cyanoacrylate } \\ \text { Speed of Sound }(\mathrm{m} / \mathrm{s}) & 1300^{a} & 1004^{b} & 3250^{a} \\ \text { Relative Dielectric Constant } & 3.72^{a} & 2.71^{c} & 2-3.5^{d} \\ \text { Resistivity }(\Omega \text {-cm) } & 10^{15 a} & 10^{15 c} & 10^{16 a, d} \\ \text { Coupling Layer thickness }(\mu \mathrm{m}) & \$ 1^{a} & \$ 1^{a} & \$ 1^{a}\end{array}$

and poor reproducibility of coupling layers can increase the spread in reflected signals (decrease PEA resolution) or add temporal offsets to the signals. Poor acoustic and electrical coupling causes enhanced reflection of the pulsed signals at interfaces and results in a diminished signal-to-noise ratio. This in turn affects the accuracy with which the pulse applied to the sample can be characterized. Other complications include polarization, relaxation effects, and incompatibility of outgassing of oils in vacuum systems.

For these reasons, it is important to understand the properties and effects of the coupling media in the PEA stack [6]. This provides the motivation for this study.

\section{COUPLING LAYERS}

Coupling layers used in this study include no coupling media, light machine oil, silicone oil, and cyanoacrylate glue. The relevant electrical and acoustic properties of these materials are listed in Table $1[8,9,12]$. Light machine oil used is All Purpose Oil (Singer brand). Silicone oil used is $100 \%$ silicone oil (MicroLubrol Type $20050 \mathrm{cSt}$ ). The glue used is cyanoacrylate (Bob Smith Industries, Super Thin Insta-Cure Cyanoacrylate, super glue). The relative dielectric constants range from 2.0-3.7 and speed of sound ranges from $1000-3250$ $\mathrm{m} / \mathrm{s}$ for the coupling media.

The thicknesses of the oils are all measured as $\lesssim 1 \mu \mathrm{m}$ thick with a micrometer. The thickness of the glue has been measured with a scanning electron microscope and thin film interference to be consistently approximately $1 \mu \mathrm{m}$ thick. Thus, interface layers are $\lesssim 1 \%$ of the test sample thicknesses and $\lesssim 10 \%$ of the sensor thickness or PEA spatial resolution.

\section{PEA MEASUREMENTS}

To compare the effects of these coupling media, measurements were made on $250 \mu \mathrm{m}$ thick polymethylmethacrylate (PMMA) samples obtained from Goodfellow [13]. Different samples and sensors were used for each test, as cleaning the samples and sensors could have caused damage. There is some variance in the thickness of the PMMA samples ( 250-270 $\mu \mathrm{m})$ and PVDF sensors ( 11-14 $\mu \mathrm{m})$ for each test, as seen with the shift in High Voltage (HV) electrode peaks in Fig. 2. The ground electrode peak is on the left and HV electrode peak on the right, for each measurement. The peak-to-peak separations in each measurement are within instrument error of the sample thicknesses (see Fig. 3). The variation in thickness is consistently to within $\lesssim 1 \%$ variation in a given sample or sensor. The waveforms were aligned according to the ground electrode peak position. Although the ideal way for comparison may be to align the signals by the initial rising edge, this is harder to achieve in practice. This is
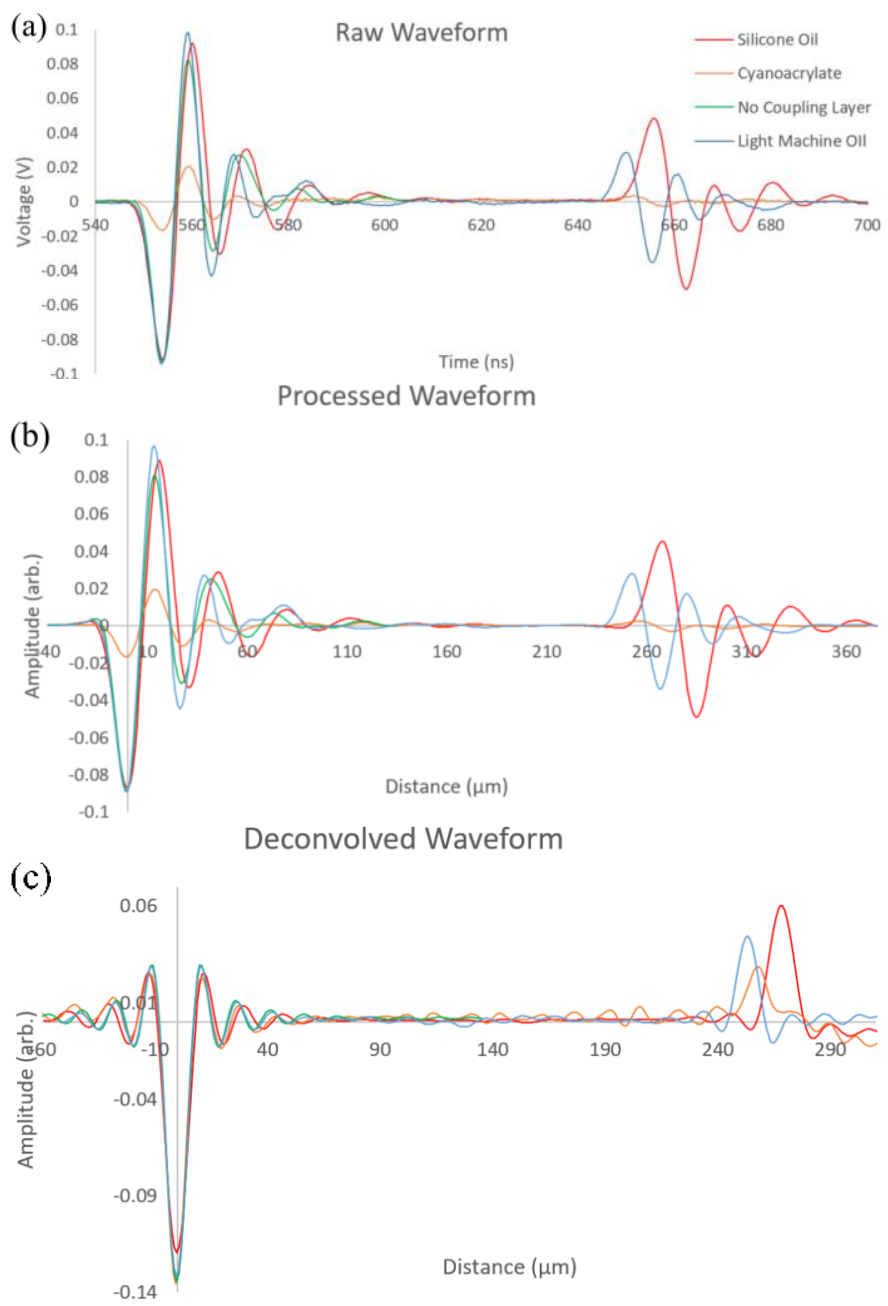

Fig. 2. Waveforms for each test are shown at each stage of signal processing. (a) Raw signal. (b) Processed waveforms have undergone a DC offset correction and a bandpass filter. Peaks shifted to zero and time converted into distance. (c) Deconvolved waveforms use a reference waveform to perform a deconvolution as a final part of the signal processing.

because the peaks broaden based on the sensor, coupling media, and applied pulse thereby shifting the peak of the response. This is an arbitrary choice and has no effect on the results. It should be noted the rising-edge-to-rising-edge separations agree with peak-to-peak measurements to within $\lesssim 1 \mathrm{~ns}$ or $\lesssim 3 \mu \mathrm{m}$.

The speed of sound for PMMA was measured to be 2630 $\mathrm{m} / \mathrm{s}$ using the difference in time between the rising edges of the ground and HV electrodes of the silicone oil waveform. This speed value agrees with literature values to within $4 \%[10,11]$.

Measurements were made with a custom ambient PEA test apparatus [1]. Pulse amplitudes of $2000 \mathrm{~V}$ and width of $5 \mathrm{~ns}$ were used. A static $8 \mathrm{kV}$ DC bias was applied across the sample to induce charge on the electrodes and near the surface of the sample. The sensors used were commercial polyvinylidene fluoride (PVDF) piezoelectric sensors (cut from film made by Measurement Specialties Inc.) with nominal $9 \mu \mathrm{m}$ thickness (measured to be 11-14 $\mu \mathrm{m}$ thick). The PVDF sensor and backing are held in place within the PEA fixture enclosure, and the sensor is clamped in place by bolting the ground electrode disc to the fixture with the sensor between the electrode and 

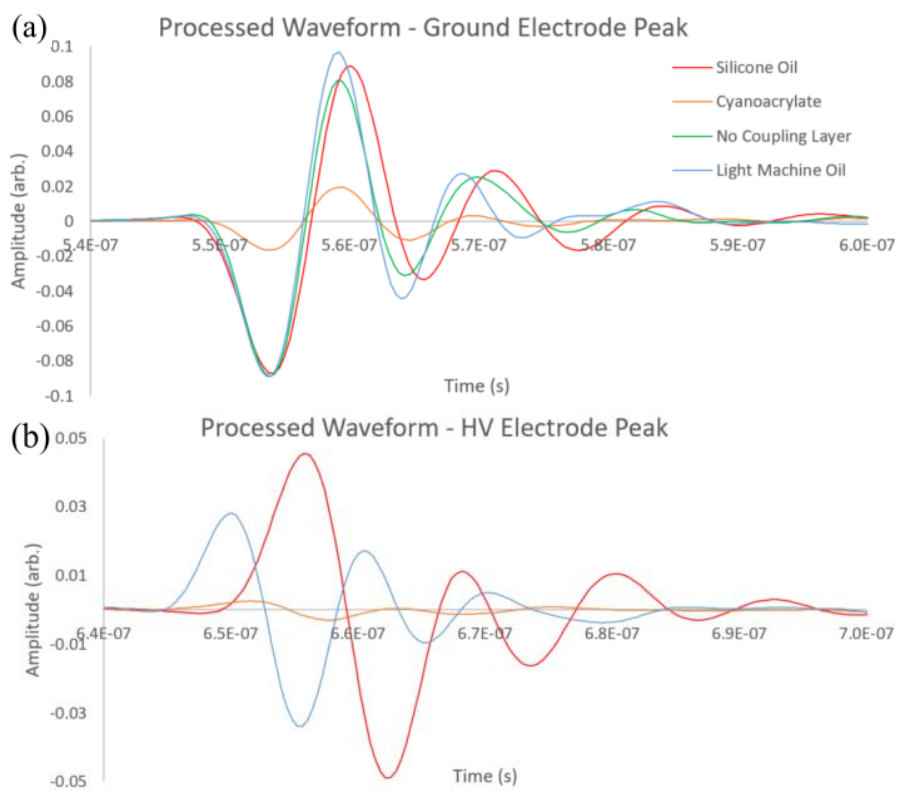

Fig. 3. Peak shifts in processed waveforms. Zoomed in look at (a) ground electrode peak and (b) HV electrode peak.

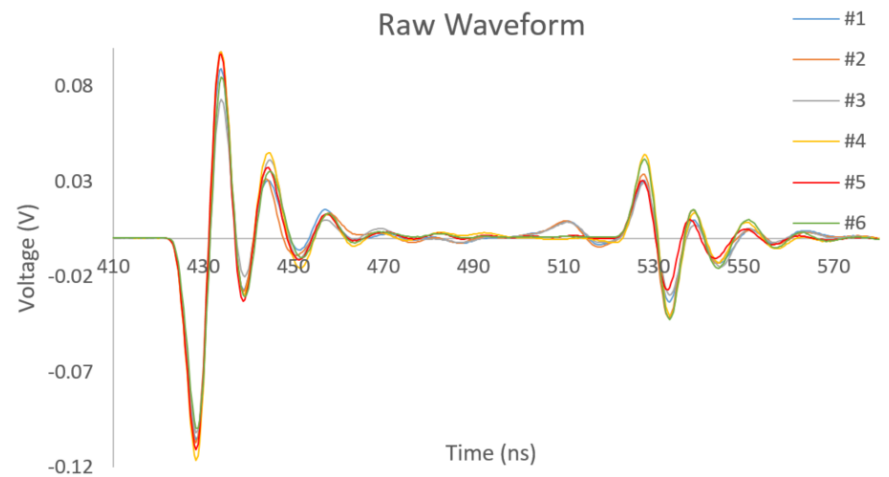

Fig. 4. Measurements are consistent and reproducible, shown above are 6 repeated PEA measurements. Charge layer near HV electrode in waveforms \#13 and near ground electrode in \#4.

backing. The HV electrode is then bolted down on top of the sample and ground electrode.

Initial measurements were made as a reference standard, with no coupling media between any surfaces. Acoustic coupling between the HV electrode and the sample is unnecessary, as its absence only inhibits observation of the charge induced on the HV electrode. For oil coupling media, oil was applied between HV (anode) electrode-sample, sampleground (cathode) electrode, ground electrode-PVDF sensor, and PVDF sensor-absorption backing interfaces. Oil was applied at each interface and wiped to minimize the coupling layer thickness. Between measurements each interface was cleaned thoroughly with isopropyl alcohol. For cyanoacrylate glue tests, only the sample-ground electrode interface had glue applied and other interfaces had no coupling media. Pressure was applied to the sample-ground electrode interface as it cured to insure a thin glue layer was formed.

Repeated measurements were made (see Fig. 4), where the PEA stack was disassembled and reassembled between measurements using the same sensor and sample, to gauge the reproducibility of the PEA system used. The same settings and sample were used to showcase reproducibility with the light machine oil measurement for this study. The shape of the waveform is consistent for each measurement with the same PVDF sensor. It should be noted that the waveform shape changed slightly if the PVDF sensor was changed [see Fig. 3(a)], specifically in the light machine oil waveform ringing. The only differences between the waveforms in Fig. 4 are a slight change in amplitude ( $\pm 8 \%$ variation) and a noticeable charge layer near the HV electrode in measurements \#1-3 and near the ground electrode in \#4. A fresh cut sample of PMMA was used for measurements \#5 and \#6 to eliminate the charge layer.

\section{ANALYSIS AND RESULTS}

PEA measurements are shown throughout the analysis process in Fig 2 [7]. "Raw" data are the data as it is measured from the oscilloscope. "Processed" data denotes that the waveform has undergone a DC offset correction as well as applying a bandpass filter. This decreases the amplitude of the signal by up to $10 \%$ in most cases, although the decrease in amplitude is $30 \%$ for the HV peak in the cyanoacrylate waveform (see Table 2). The spatial resolution consistently increased by up to $10 \%$ with processing. The shape of the signal stays substantially the same, although the noise is reduced and a slight overshoot is noticed near the peaks.

"Deconvolved" data denotes that a deconvolution using a reference waveform has been performed. The reference waveform used is the ground electrode peak unless there is charge within the sample, which is not the case for this study. This rescales the amplitude, and drastically improves the overall shape/quality of the signal. This indicates that low amplitude signals are not necessarily worse than higher amplitude signals, as they are rescaled after signal processing. What is important is not the absolute amplitudes but the signalto-noise and the ratio of the HV-to-ground electrode peaks. The spatial resolution increases again with deconvolution from the processed waveform by up to $30 \%$. Overall, from raw to deconvolved data the waveforms increase spatial resolution 10$20 \%$ for the oils and $20-40 \%$ for the cyanoacrylate glue.

The amplitude of the initial ground electrode peaks and HV peaks were determined and the ratios were calculated (see Table 2). The ratios of these peaks indicates the level of attenuation in the sample, as well as the efficiency and quality of acoustic coupling and the relationship between the acoustic properties of each layer. This is because, with attenuation accounted for, the signal from the HV peak has to traverse every interface in the PEA stack while the signal from the ground electrode peak only has to pass through the interface to get to the PVDF sensor.

The Full Width at Half Max (FWHM) of the peaks, are used as a measure of the spatial resolution. The FWHM in time is multiplied by the measured speed of sound in PMMA (2630 $\mathrm{m} / \mathrm{s}$ ) to obtain the approximate spatial resolution for the measurement.

Peak ratios of $\mathrm{HV} /$ ground electrode peaks are consistent throughout the analysis process for each coupling media. They 
Table 2. PEA Results.

$\begin{array}{lcccc} & \text { No Oil } & \text { Light Machine Oil } & \text { Silicone Oil } & \text { Cyanoacryla } \\ \text { Raw } & & & & \\ \text { Ground Peak Ampl. }(\mathrm{mV}) & 93.1 & 94.0 & 91.3 & 16.8 \\ \text { HV Peak Ampl. }(\mathrm{mV}) & n / a & 28.8 & 48.7 & 3.7 \\ \text { Ratio of HV/Ground Peaks } & n / a & 0.306 & 0.532 & 0.220 \\ \text { Peak-to-Peak (ns) } & n / a & 96.3 & 102 & 97.6 \\ \text { Peak-to-Peak }(\mu \mathrm{m}) & n / a & 253 & 268 & 257 \\ \text { FWHM Ground Peak }(\mu \mathrm{m}) & 11.3 & 11.3 & 12.9 & 11.0 \\ \text { FWHM HV Peak }(\mu \mathrm{m}) & n / a & 11.3 & 14.1 & 15.7 \\ \text { Processed } & & & & \\ \text { Ground Peak Ampl. }(\mathrm{mV}) & 88.9 & 89.1 & 86.8 & 16.6 \\ \text { HV Peak Ampl. }(\mathrm{mV}) & n / a & 28.2 & 45.6 & 2.60 \\ \text { Ratio of HV/Ground Peaks } & n / a & 0.317 & 0.525 & 0.157 \\ \text { Peak-to-Peak }(\mathrm{ns}) & n / a & 96.3 & 101.8 & 97.6 \\ \text { Peak-to-Peak }(\mu \mathrm{m}) & n / a & 253 & 268 & 257 \\ \text { FWHM Ground Peak }(\mu \mathrm{m}) & 11.0 & 11.0 & 12.5 & 10.8 \\ \text { FWHM HV Peak }(\mu \mathrm{m}) & n / a & 11.1 & 13.5 & 14.4 \\ \text { Deconvolved } & & & & \\ \text { Ground Peak Ampl. }(\mathrm{mV}) & 134.2 & 132 & 120 & 135 \\ \text { HV Peak Ampl. }(\mathrm{mV}) & n / a & 44.5 & 60.3 & 28.8 \\ \text { Ratio of HV/Ground Peaks } & n / a & 0.338 & 0.504 & 0.213 \\ \text { Peak-to-Peak }(\mathrm{ns}) & n / a & 96.9 & 102 & 98.3 \\ \text { Peak-to-Peak }(\mu \mathrm{m}) & n / a & 255 & 268 & 259 \\ \text { FWHM Ground Peak }(\mu \mathrm{m}) & 9.13 & 9.32 & 10.3 & 8.85 \\ \text { FWHM HV Peak }(\mu \mathrm{m}) & n / a & 9.70 & 12.2 & 10.3 \\ & & & & \end{array}$

are $30 \%, 50 \%$, and $20 \%$ for light machine oil, silicone oil, and cyanoacrylate, respectively. The low ratio for the cyanoacrylate is expected as there is no coupling layer at the HV electrodesample interface. The silicone oil has the highest peak ratio suggesting that it is the most efficient acoustic coupling media. The spatial resolution, as based on ground electrode peak, is noticeably worse (10-30\%) for the silicone oil compared to the other three measurements. Note that the cyanoacrylate is not necessarily less efficient as there is no cyanoacrylate at the HV electrode-sample interface, so the peak ratio for that measurement is less meaningful.

The FWHM of the ground electrode peak is $\sim 11 \mu \mathrm{m}$ for the raw waveforms with no coupling media, light machine oil, and cyanoacrylate, but $17 \%$ worse for silicone oil at $13 \mu \mathrm{m}$. The HV electrode peak amplitude is the same for all but the cyanoacrylate, which is expectedly worse by $45 \%$ because of the lack of coupling at the HV electrode interface. The processed waveforms show the same trends. After deconvolution, the cyanoacrylate spatial resolution is the best by a $3 \%$ margin over the no coupling media, which is within error. Silicone oil has the worst resolution, differing by $16 \%$ from cyanoacrylate. The spatial resolution after signal processing is $\sim 9 \mu \mathrm{m}$ for all but the silicone oil which is $\sim 10 \mu \mathrm{m}$, for the ground electrode peak. The HV electrode resolution for the cyanoacrylate starts out as the worst resolution, but after deconvolution, it is better than the silicone oil at 10.3, 9.7, and $12.2 \mu \mathrm{m}$ for cyanoacrylate, light machine oil, and silicone oil, respectively. Overall, the silicone oil has about $18 \%$ worse spatial resolution after signal processing than the other coupling medias.

\section{COnClusions}

Results from this study allow specific conclusions to be drawn for each of the four coupling media.

No coupling media is a viable option if the HV electrode is directly in contact with the sample. Amplitudes of the light machine oil, silicone oil, and no coupling media were of similar amplitude to well within normal variation. Note, with systems requiring irradiation in vacuo the $\mathrm{HV}$ electrode is not placed directly in contact with the sample [6].

The silicone oil had the worst spatial resolution, which was worse by $18 \%$. Silicone oil was the most efficient at propagating acoustic signals. It should be noted that there are problems with polarization of silicone oil under applied field that are not directly discussed or studied in this paper. Silicone oil should be avoided if possible.

Light machine oil is a viable option for ambient systems, as the spatial resolution and amplitudes are better than silicone oil and comparable to the other coupling medias. This is however not an option for in vacuo systems.

Despite having a lower amplitude signal, cyanoacrylate on the single surface between the ground electrode and sample, with no coupling oil anywhere else, marginally has the best spatial resolution after signal processing. The amplitude of the signal is comparable to the other coupling medias after deconvolution, as they are rescaled. The signal-to-noise and raw data amplitude could potentially be increased with the addition of light machine oil applied to the PVDF sensor interfaces, which in our custom system is outside vacuum and not changed between samples.

In an in vacuo PEA system, having a single glued interface is a viable and encouraged option, as it has the best resolution (11 $\mu \mathrm{m}$ raw, and $9 \mu \mathrm{m}$ after signal processing), has good signalto-noise properties, has decent $\mathrm{HV}$ /ground electrode peak ratios, and is the only vacuum compatible coupling media tested.

\section{ACKNOWLEDGMENTS}

Research was supported by an AFRL STTR award through Box Elder Innovations and a USU Presidential Doctoral Research Fellowship.

\section{REFERENCES}

[1] J. R. Dennison and L. H. Pearson, "Pulse Electro-Acoustic (PEA) Measurements of Embedded Charge Distributions," Proc. SPIE Optics and Photonics Conf., 8876, 887612-1-887612-11, (2013)

[2] T. Maeno, et. al., "Measurement of Spatial Charge Distribution in Thick Dielectrics Using the Pulsed Electroacoustic Method," IEEE Trans. Electrical Insulation, 23(3):433, (1988)

[3] M. Arnaout, L. Berquez, F. Baudoin, and D. Payan, "Contribution to Improving the Spatial Resolution of a Pulsed Electro Acoustic Cell Measurement," IEEE Int. Conf. Solid Dielectrics, Germany, (2010).

[4] V. Griseri, et. al., "Space-Charge Detection and Behavior Analysis in Electronirradiated Polymers," IEEE Trans. Plasma Sci., 34(5):2185, (2006).

[5] M. Andrade, et. al., "Different Space Charge Behavior of Materials Used in AC and DC Systems", IEEE Conference on Electrical Insulation and Dielectric Phenomenon, Forth Worth, TX, (2017)

[6] V. Griseri, et. al., "Assessment of measuring conditions with the pulse electroacoustic system adapted to work under electronic irradiation", 2003 Annual Report Conference on Electrical Insulation and Dielectric Phenomena. (2003)

[7] L. H. Pearson, et. al., "PEA System Modeling and Signal Processing for Measurement of Volume Charge Distributinos in Thin Dielectric Films,", IEEE Trans. Plasma Sci., 45(8):1955, (2017)

[8] LOCTITE Cyanoacrylate Technical Data Sheet, LOCTITE 495, (2004)

[9] "Dielectric Properties of Pure Silicone Fluids", Clearco Products Inc.

[10] T. Takada, et.al. 2006. Space Charge Measurement in Dielectrics and Insulating Materials. CIGRE Task Force D1.12.01-288. pp. 1-29.

[11] P.E. Bloomfield, et. al., "Experimental Study of the Acoustical Properties of Polymers Utilized to Construct PVDF Ultrasonic Transducers and the AcoustoElectric Properties of PVDF and P(VDF/TrFE) Films". IEEE Trans. Ultrasonics, Ferroelectrics and Frequency Control, 47(6):1398 (2000)

[12] A. Schröder and E. Raphael 1992 EPL 17565

[13] Goodfellow, "Polymethylmethacrylate - Sheet, PMMA, Acrylc" ME303005, https://www.goodfellowusa.com/catalog/GFCat4J.php?ewd_token=KjfxPRb9qal ol8q7vp28jJU3U3HWvW\&n=PEbzQbZBQ16yF5XxGUqZyloe0HRznU (2018) 
